PROCEEDINGS OF THE

AMERICAN MATHEMATICAL SOCIETY

Volume 139, Number 8, August 2011, Pages 2821-2826

S 0002-9939(2011)10733-0

Article electronically published on February 8, 2011

\title{
RESONANCE FOR THE ISOTHERMAL SYSTEM OF ISENTROPIC GAS DYNAMICS
}

\author{
YUN-GUANG LU
}

(Communicated by Matthew J. Gursky)

\begin{abstract}
In this paper, we remove the restriction $z_{0}(x) \geq 0$ or $w_{0}(x) \leq 0$ in the paper "Existence of Solutions to Hyperbolic Conservation Laws with a Source" (Commun. Math. Phys., 187 (1997), 327-340) and obtain the existence of solutions for the resonant, isothermal system of isentropic gas dynamics.
\end{abstract}

\section{Introduction}

Consider

$$
\left\{\begin{array}{l}
\rho_{t}+(\rho u)_{x}=-\frac{a^{\prime}(x)}{a(x)} \rho u, \\
(\rho u)_{t}+\left(\rho u^{2}+P(\rho)\right)_{x}=-\frac{a^{\prime}(x)}{a(x)} \rho u^{2},
\end{array}\right.
$$

with bounded initial data

$$
(\rho(x, 0), u(x, 0))=\left(\rho_{0}(x), u_{0}(x)\right), \quad \rho_{0}(x) \geq 0,
$$

where $\rho$ is the density of gas, $u$ the velocity, $P=P(\rho)$ the pressure, and $a(x)$ represents the cross-sectional area of a variable duct. System (1.1) is of interest because resonance occurs. This means there is a coincidence of wave speeds from different families of waves (see [EGM, GMP, IT, Liu] and the references cited therein for the details).

By simple calculations, two eigenvalues of system (1.1) are

$$
\lambda_{1}=\frac{m}{\rho}-\sqrt{P^{\prime}(\rho)}, \quad \lambda_{2}=\frac{m}{\rho}+\sqrt{P^{\prime}(\rho)}
$$

with corresponding Riemann invariants

$$
z(u, v)=\int_{0}^{\rho} \frac{\sqrt{P^{\prime}(s)}}{s} d s-\frac{m}{\rho}, \quad w(u, v)=\int_{0}^{\rho} \frac{\sqrt{P^{\prime}(s)}}{s} d s+\frac{m}{\rho},
$$

where $m=\rho u$.

In the paper [KL, the authors first studied the global existence of weak solutions for the Cauchy problem (1.1)-(1.2) by using the vanishing viscosity method coupled with the compensated compactness theory. Since the super-linear source term is

Received by the editors April 6, 2010 and, in revised form, April 27, 2010 and July 26, 2010. 2010 Mathematics Subject Classification. Primary 35L65, 76N10.

Key words and phrases. Resonance, gas dynamics, global weak solution, $L^{\infty}$ estimate.

The author thanks the referee for many valuable suggestions.

(C)2011 American Mathematical Society Reverts to public domain 28 years from publication 
in the second equation in (1.1), when we prove the global existence, the main difficulty is to obtain $L^{\infty}$ estimates, of viscosity solutions, independent of the viscosity perturbation constant $\varepsilon$. With the help of the condition $z_{0}(x) \leq 0$ or $w_{0}(x) \leq 0$, the $L^{\infty}$ bound of $\left(\rho^{\varepsilon}, m^{\varepsilon}\right)$ was obtained in $\mathrm{KL}$ by using the maximum principle for the polytropic gas $P(\rho)=\frac{1}{\gamma} \rho^{\gamma}, \gamma>1$.

Without the condition $z_{0}(x) \leq 0$ or $w_{0}(x) \leq 0$, many authors tried to prove the same uniform estimate for the spherically, symmetric solutions in the region of $x>1$, where $a(x)=x^{2}$. However, as pointed out in the paper [Ts1], there are some defects in the proofs in these papers. Instead of the uniform estimate, a reasonable estimate depending on the variable $x: z(x, t) \leq C x^{-\frac{2(\gamma-1)}{\gamma+1}}, C>0$, was obtained in Ts1] for $1<\gamma<\frac{5}{3}$.

Since the proof of $\gamma=1$ is different from that of $\gamma>1$, in this paper we first study the isothermal case $P(\rho)=\rho$ and prove the global existence of weak solutions for the Cauchy problem (1.1)-(1.2) for general bounded initial data. The main result is given in the following:

Theorem 1.1. Let $P(\rho)=\rho, 0<a_{L} \leq a(x) \leq M$ for $x$ in any compact set $x \in(-L, L), A(x)=-\frac{a^{\prime}(x)}{a(x)} \in C(R)$ and $A^{\prime}(x)$ exist. Moreover, $|A(x)| \leq M, 0 \leq$ $A^{\prime}(x) \leq A_{L}$, where $M$ is a positive constant and $a_{L}, A_{L}$ are positive constants depending on $L$. Then the Cauchy problem (1.1)-(1.2) has a bounded weak solution $(\rho, u)$ which satisfies system (1.1) in the sense of distributions and

$$
\int_{0}^{\infty} \int_{-\infty}^{\infty} \eta(\rho, m) \phi_{t}+q(\rho, m) \phi_{x}+A(x)\left(\eta(\rho, m)_{\rho} \rho u+\eta(\rho, m)_{m} \rho u^{2}\right) \phi d x d t \geq 0,
$$

where $(\eta, q)$ is a pair of entropy-entropy flux of system (1.1), $\eta$ is convex, and $\phi \in C_{0}^{\infty}\left(R \times R^{+}-\{t=0\}\right)$ is a positive function.

Example 1.2. For a small constant $\varepsilon_{0}>0$, we choose a continuous function $A(x)$ to approximate $-\frac{2}{x}$ :

$$
A(x)=\left\{\begin{array}{l}
0, \quad \text { for } \quad x \geq \frac{2}{\varepsilon_{0}} \\
-4 \varepsilon_{0}+2 \varepsilon_{0}^{2} x, \quad \text { for } \quad \frac{1}{\varepsilon_{0}} \leq x \leq \frac{2}{\varepsilon_{0}} \\
-\frac{2}{x}, \quad \text { for } \quad \varepsilon_{0} \leq x \leq \frac{1}{\varepsilon_{0}} \\
-\frac{2 e^{\frac{1}{2}}}{\varepsilon_{0}} e^{-\frac{x^{2}}{2 \varepsilon_{0}^{2}}}, \quad \text { for } \quad 0 \leq x \leq \varepsilon_{0} \\
-\frac{2 e^{\frac{1}{2}}}{\varepsilon_{0}}, \quad \text { for } \quad x \leq 0
\end{array}\right.
$$

then

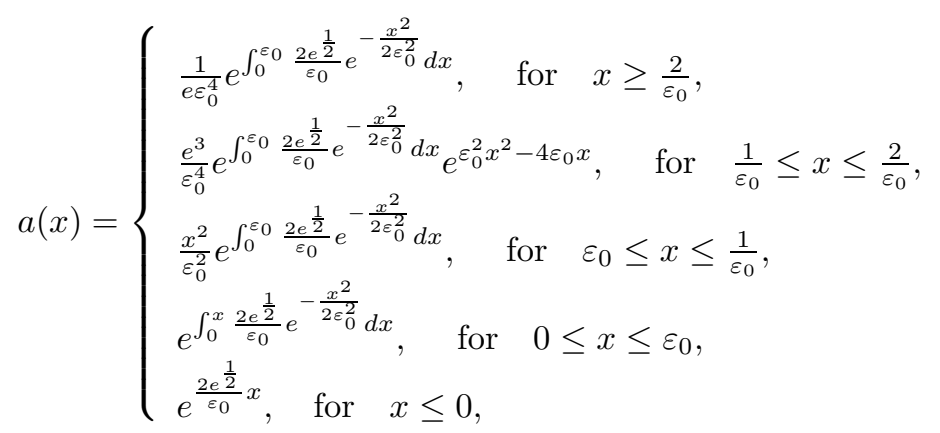

and all conditions in Theorem 1.1 are satisfied. 
Remark 1.3. The global existence of symmetrical weak solutions of the isothermal gas dynamics system (1.1) in the Lagrangian coordinates was well studied in [s2, MMU] by using the Glimm scheme method [Gl, Ni].

Remark 1.4. The homogeneous case of isothermal system (1.1) $\left(a^{\prime}(x)=0\right)$ in the Euler coordinates was studied in $[\mathrm{HW}$ ] by using the compensated compactness theory [Di, Ta] (see also $\mathrm{Lu}$ ] for the details).

\section{Proof of Theorem 1.1}

Let $v=\rho a^{\delta}(x)$, then we may rewrite (1.1) as

$$
\left\{\begin{array}{l}
v_{t}+(v u)_{x}=0 \\
(v u)_{t}+\left(v u^{2}+v\right)_{x}+A^{\delta}(x) v=0
\end{array}\right.
$$

where $A^{\delta}(x)=-\frac{a^{\delta}(x)^{\prime}}{a^{\delta}(x)}$ is given in (2.6). The two eigenvalues of (2.1) are

$$
\lambda_{1}=u-1, \quad \lambda_{2}=u+1
$$

with corresponding Riemanna invariants

$$
z(v, m)=\ln v-\frac{m}{v}, \quad w(v, m)=\ln v+\frac{m}{v}, \quad m=v u .
$$

Now we consider the Cauchy problem for the related parabolic system

$$
\left\{\begin{array}{l}
v_{t}+(v u)_{x}=\varepsilon v_{x x}, \\
(v u)_{t}+\left(v u^{2}+v\right)_{x}+A^{\delta}(x) v=\varepsilon(v u)_{x x},
\end{array}\right.
$$

with initial data

$$
(v(x, 0), v(x, 0) u(x, 0))=\left(v_{0}^{\delta}(x), v_{0}^{\delta}(x) u_{0}^{\delta}(x)\right),
$$

where

$$
\left(v_{0}^{\delta}(x), u_{0}^{\delta}(x), A^{\delta}(x)\right)=\left(a(x) \rho_{0}(x)+\delta, u_{0}(x), A(x)\right) * G^{\delta}
$$

and $G^{\delta}$ is a mollifier. Then

$$
\left(v_{0}^{\delta}(x), u_{0}^{\delta}(x), A^{\delta}(x)\right) \in C^{\infty}(R) \times C^{\infty}(R) \times C^{\infty}(R)
$$

and

$$
\begin{aligned}
& \left.v_{0}^{\delta}(x) \geq \delta, \quad v_{0}^{\delta}(x)+\mid u_{0}^{\delta}(x)\right)|\leq M, \quad| A^{\delta}(x) \mid \leq M, \quad 0 \leq A^{\delta}(x) \leq A_{L}, \\
& \begin{cases}\delta\left|u_{0, x}^{\delta}(x)\right| \leq M, \\
\left|v_{0, x}^{\delta}(x)\right| \leq M(\delta), \quad\left|u_{0, x}^{\delta}(x)\right| \leq M(\delta), \\
\left|v_{0, x x}^{\delta}(x)\right| \leq M(\delta), \quad\left|u_{0, x x}^{\delta}(x)\right| \leq M(\delta) .\end{cases}
\end{aligned}
$$

We now use the maximum principle to estimate the viscosity solutions of (2.4) and (2.5). We multiply (2.4) by $\left(w_{v}, w_{m}\right)$ and $\left(z_{v}, z_{m}\right)$, respectively, to obtain

$$
\begin{aligned}
z_{t}+\lambda_{1} z_{x}-A^{\delta}(x)= & \varepsilon z_{x x}-\varepsilon\left(z_{v v} v_{x}^{2}+2 z_{v m} v_{x} m_{x}+z_{m m} m_{x}^{2}\right) \\
& =\varepsilon z_{x x}+\frac{2 \varepsilon}{v} v_{x} z_{x}-\frac{\varepsilon v_{x}^{2}}{v^{2}} \\
& \leq \varepsilon z_{x x}+\frac{2 \varepsilon}{v} v_{x} z_{x}
\end{aligned}
$$


and

$$
\begin{aligned}
w_{t}+\lambda_{2} w_{x}+A^{\delta}(x) & =\varepsilon w_{x x}-\varepsilon\left(w_{v v} v_{x}^{2}+2 w_{v m} v_{x} m_{x}+w_{m m} m_{x}^{2}\right) \\
& =\varepsilon w_{x x}+\frac{2 \varepsilon}{v} v_{x} w_{x}-\frac{\varepsilon v_{x}^{2}}{v^{2}} \\
& \leq \varepsilon w_{x x}+\frac{2 \varepsilon}{v} v_{x} w_{x} .
\end{aligned}
$$

Letting $z=\bar{z}+M t, w=\bar{w}+M t$, where $M$ is the bound of $\left|A^{\delta}(x)\right|$, we have from (2.9) -(2.10) that

$$
\bar{z}_{t}+\lambda_{1} \bar{z}_{x} \leq \varepsilon \bar{z}_{x x}+\frac{2 \varepsilon}{v} v_{x} \bar{z}_{x}
$$

and

$$
\bar{w}_{t}+\lambda_{2} \bar{w}_{x} \leq \varepsilon \bar{w}_{x x}+\frac{2 \varepsilon}{v} v_{x} \bar{w}_{x} .
$$

Using the maximum principle to (2.11)-(2.12), we have the estimates

$$
\bar{z}\left(v^{\varepsilon}, m^{\varepsilon}\right) \leq C_{1}, \quad \bar{w}\left(v^{\varepsilon}, m^{\varepsilon}\right) \leq C_{2}
$$

or

$$
z\left(v^{\varepsilon}, m^{\varepsilon}\right) \leq C_{1}+M t=M(t), \quad w\left(v^{\varepsilon}, m^{\varepsilon}\right) \leq C_{2}+M t=M(t),
$$

where $C_{1}, C_{2}$ are two positive constants depending only on the bounds of the initial data and $a(x)$. Since the initial data $v_{0}^{\delta}(x) \geq \delta$, the local solution $v^{\varepsilon}>0$. The estimates in (2.13) give us the following $L^{\infty}$ bound:

$$
v^{\varepsilon} \leq M(t), \quad \ln v^{\varepsilon}-M(t) \leq u^{\varepsilon} \leq M(t)-\ln v^{\varepsilon}, \quad\left|m^{\varepsilon}\right| \leq M(t),
$$

where $M(t)$ is independent of $\varepsilon$.

To estimate the positive, lower bound of $v^{\varepsilon}$, we substitute the first equation in (2.4) into the second to obtain

$$
u_{t}+u u_{x}+\frac{1}{v} v_{x}+A^{\delta}(x)=\varepsilon u_{x x}+2 \varepsilon(\ln v)_{x} u_{x} .
$$

Differentiating (2.15) with respect to $x$, we have

$$
\left(u_{x}\right)_{t}+u_{x}^{2}+u u_{x x}+(\ln v)_{x x}+A^{\delta}(x)^{\prime}=\varepsilon\left(u_{x}\right)_{x x}+2 \varepsilon(\ln v)_{x x} u_{x}+2 \varepsilon(\ln v)_{x} u_{x x} .
$$

Let

$$
\theta=u_{x}-\frac{1}{2 \varepsilon}
$$

then

$$
\theta_{t}+\left(u-2 \varepsilon(\ln v)_{x}\right) \theta_{x}-2 \varepsilon(\ln v)_{x x} \theta+u_{x}^{2}+A^{\delta}(x)^{\prime}=\varepsilon \theta_{x x} .
$$

Since $A^{\delta}(x)^{\prime} \geq 0$, we have the following inequality from (2.18):

$$
\theta_{t}+\left(u-2 \varepsilon(\ln v)_{x}\right) \theta_{x}-2 \varepsilon(\ln v)_{x x} \theta \leq \varepsilon \theta_{x x} .
$$

Then we may apply the maximum principle to (2.19) to obtain

$$
\theta \leq 0 \quad \text { or } u_{x} \leq \frac{1}{2 \varepsilon}
$$

if we let $\delta$ and $\varepsilon$ satisfy the relation

$$
2 \varepsilon M \leq \delta
$$

where $M$ is given in (2.8). 
In fact, let

$$
\theta=\left(q+\frac{N\left(x^{2}+c L e^{t}\right)}{L^{2}}\right) e^{h t}
$$

where $c, h$ and $N$ are positive constants and $c>2|a(x, t)|+2 \varepsilon$ in $R \times[0, T], N$ is an upper bound for $\theta$ (local solution) and $h$ a bound for $b(x, t)$ on $R \times[0, T]$ and the functions $a(x, t), b(x, t)$ are given as follows:

$$
a(x, t)=u-2 \varepsilon(\ln v)_{x}, \quad b(x, t)=-2 \varepsilon(\ln v)_{x x} .
$$

The function $q$, as is easily seen, satisfies the inequality

$$
\begin{aligned}
q_{t}+\frac{c N L e^{t}}{L^{2}} e^{h t}+ & h\left(q+\frac{N\left(x^{2}+c L e^{t}\right)}{L^{2}}\right)+a(x, t)\left(q_{x}+\frac{2 N x}{L^{2}}\right) \\
& +b(x, t)\left(q+\frac{N\left(x^{2}+c L e^{t}\right)}{L^{2}}\right) \leq \varepsilon q_{x x}+\frac{2 N \varepsilon}{L^{2}},
\end{aligned}
$$

resulting from (2.19). Moreover

$$
q(x, 0)<0, \quad q(+L, t)<0, \quad q(-L, t)<0 .
$$

Then using the standard maximum principle we see from (2.23) and (2.24) that $q \leq 0$ on $(x, t) \in(-L, L) \times[0, T]$. For any fixed $(x, t)$, letting $L \uparrow \infty$ in (2.22), we get $\theta \leq 0$.

Using the first equation in (2.4) and the estimate (2.21), we have

$$
v^{\varepsilon} \geq \delta e^{-\frac{t}{2 \varepsilon}},
$$

since $v_{0}^{\varepsilon}(x) \geq \delta$. By applying the general contracting mapping principle to an integral representation of (2.4), we can obtain a local solution of the Cauchy problem (2.4)-(2.5) first. Then using the lower, positive estimate (2.25) and the $L^{\infty}$ estimates given in (2.14), we can obtain the existence and uniqueness of a smooth solution of the Cauchy problem (2.4)-(2.5) (the details can be found in DW] or Di ). Finally, applying the convergence frame given in $\mathrm{HW}$ we have the pointwise convergence

$$
\left(v^{\varepsilon, \delta}(x, t), m^{\varepsilon, \delta}(x, t)\right) \rightarrow(v(x, t), m(x, t)) \text { a.e., as } \varepsilon, \delta \rightarrow 0
$$

or

$$
\left(\rho^{\varepsilon, \delta}(x, t),\left(\rho^{\varepsilon, \delta} u^{\varepsilon, \delta}\right)(x, t)\right) \rightarrow(\rho(x, t),(\rho u)(x, t)) \text { a.e., as } \varepsilon, \delta \rightarrow 0 .
$$

We rewrite (2.4) as

$$
\left\{\begin{array}{l}
\rho_{t}+(\rho u)_{x}=-\frac{a^{\prime}(x)}{a(x)} \rho u+\varepsilon \rho_{x x}+2 \varepsilon \frac{a^{\prime}(x)}{a(x)} \rho_{x}+\varepsilon \frac{a^{\prime \prime}(x)}{a(x)} \rho, \\
(\rho u)_{t}+\left(\rho u^{2}+\rho\right)_{x}=-\frac{a^{\prime}(x)}{a(x)} \rho u^{2}+\varepsilon(\rho u)_{x x}+2 \varepsilon \frac{a^{\prime}(x)}{a(x)}(\rho u)_{x}+\varepsilon \frac{a^{\prime \prime}(x)}{a(x)}(\rho u),
\end{array}\right.
$$

where, for simplicity, the indexes $\varepsilon, \delta$ are dropped. Since $\frac{a^{\prime}(x)}{a(x)}$ is bounded for all $x \in R$ and $\frac{a^{\prime \prime}(x)}{a(x)}$ is bounded for $x$ in any compact support set $(-L, L)$, then multiplying a suitable test function $\phi$ to system (2.28), we can prove that the limit $(\rho(x, t), u(x, t))$ in (2.27) satisfies system (1.1) in the sense of distributions and the Lax entropy condition (1.5). So, we complete the proof of Theorem 1.1. 


\section{REFERENCES}

[Di] R. J. DiPerna, Convergence of the viscosity method for isentropic gas dynamics, Commun. Math. Phys., 91 (1983), 1-30. MR719807 (85i:35118)

[DW] X.-X. Ding and J.-H. Wang, Global solutions for a semilinear parabolic system, Acta Math. Sci., 3 (1983), 397-414. MR812546 (86k:35058)

[EGM] P. Embid, J. Goodman and A. Majda, Multiple steady states for 1-d transsonic flow, SIAM J. Sci. and Stat. Comput., 5 (1984), 21-41. MR731879 (86a:76029)

[Gl] J. Glimm, Solutions in the large for nonlinear hyperbolic systems of equations, Comm. Pure Appl. Math., 18 (1965), 95-105. MR0194770 (33:2976)

[GMP] J. Glimm, G. Marshall and B. Plohr, A generalized Riemann problem for quasi-onedimensional gas flows, Adv. Appl. Math., 5 (1984), 1-30. MR736548 (85e:76041)

[HW] F.-M Huang and Z. Wang, Convergence of viscosity solutions for isentropic gas dynamics, SIAM J. Math. Anal., 34 (2003), 595-610. (1984), 1-30.

[IT] E. Isaacson and B. Temple, Nonlinear resonance in systems of conservation laws, SIAM J. Appl. Math., 52 (1992), 1260-1278. MR.1182123 (93f:35140)

[KL] C. Klingenberg and Y.-G. Lu, Existence of solutions to hyperbolic conservation laws with a source, Commun. Math. Phys., 187 (1997), 327-340. MR.1463831 (98d:35139)

[Liu] T.P. Liu, Resonance for a quasilinear hyperbolic equation, J. Math. Phys., 28 (1987), 2593-2602. MR913412 (88k:35122)

[Lu] Y.-G. Lu, Hyperbolic Conservation Laws and the Compensated Compactness Method, Vol. 128, Chapman and Hall, CRC Press, New York, 2002.

[MMU] T. Makino, K. Mizohata and S. Ukai, The global weak solutions of the compressible Euler equation with spherical symmetry (I), (II), Japan J. Indust. Appl. Math., 9 (1992), 431449, 11 (1994), 417-426. MR1189949 (93k:35205); MR1299954 (95j:35172)

[Ni] T. Nishida, Global solution for an initial-boundary-value problem of a quasilinear hyperbolic system, Proc. Jap. Acad., 44 (1968), 642-646. MR0236526 (38:4821)

[Ta] T. Tartar, Compensated compactness and applications to partial differential equations, In: Research Notes in Mathematics, Nonlinear Analysis and Mechanics, Heriot-Watt symposium, Vol. 4, ed. R. J. Knops, Pitman Press, London, 1979. MR584398|(81m:35014)

[Ts1] N. Tsuge, Global $L^{\infty}$ solutions of the compressible Euler equations with spherical symmetry, J. Math. Kyoto Univ., 46 (2006), 457-524. MR2311356 (2009d:35267)

[Ts2] N. Tsuge, The compressible Euler equations for an isothermal gas with spherical symmetry, J. Math. Kyoto Univ., 43 (2004), 737-754. MR2030796 (2004i:76158)

Department of Mathematics, Hangzhou Normal University, Hangzhou, 310036, People's Republic of China - and - Department of Mathematics, National University of Colombia, Bogota, Colombia

E-mail address: yglu_2000@yahoo.com 